\title{
Four Year Follow Up of Immediate Provizionalization of Osseointegrated Dental Implant in Patient with Rheumatoid Polyarthritis
}

\section{Rym Masmoudi, Hela Jegham*, Hend Ouertani and Mohamed Bassem Khattech}

Department of Medicine and Dental Surgery, Military Hospital, Montfleury, Tunis, Tunisia

*Corresponding Author: Hela Jegham, Professor, Department of Medicine and Dental Surgery, Military Hospital, Montfleury, Tunis, Tunisia.
Received: August 24, 2020

Published: September 23, 2020

(C) All rights are reserved by Hela Jegham., et al.

\begin{abstract}
Introduction: Successful osseointegration and long term maintenance of endoosseous dental implant are influenced by implant to bone interface requiring continual bone remodeling. Many factors can influence bone remodeling and lead to osseointegration failure. Rheumatoid polyarthritis (RA) is a systemic autoimmune disease characterized by bone damage. Anti-rheumatic drugs such as Methotrexate and Glycocorticoid have deleterious effects on bone metabolism and turnover.

Aim: The aim of this report is to answer to the question whether RA and anti-rheumatic drugs can be a real contraindication for implant treatment.

Observation: A lady was referred for the replacement of the teeth $\mathrm{N}^{\circ} 14$ lost after root fracture. The patient history revealed a Rheumatoid Arthritis diagnosed since 2 years. We decide to suggest her for an implant with immediate provisional crown. The crown was not loaded at the day of surgery. Six months later, the definitive crown was cemented. The implant fulfilled osseointegration clinically and at X-Rays. After 4 years we noticed marginal bone stability.

Conclusion: Although several animal and in vitro studies recorded a negative effect of anti-rheumatic drugs on bone healing many clinical reports showed that patient with RA can be successfully treated with implants but we still need more clinical studies to reinforce our findings.
\end{abstract}

Keywords: Dental Implants; Rheumatoid Arthritis; Osseointegration; Methotrexate; Glucocorticosteroids

\section{Introduction}

In the last three decades implants have been used for the rehabilitation of partially and fully edentulous patients with much success. The literature reported success rates reaching 90 to $95 \%$. The success of dental implants depends on osseointegration defined as a direct contact on the light microscopic level between living bone tissue and an implant [1].

The understanding of mechanisms of osseointegration confirmed that it is similar to the bone remodeling and its repair [2] including inflammation, osteoblasts attachment to the implant surface and bone remodeling process. The last stage is the forma- tion of a close relation between bone and implant connected by collagenous filaments providing the long term function of dental implant [3].

Osseointegration may be affected by some factors such as implant design and characteristics, surface properties, anatomic location, implant bed preparation, bone quality and quantity, systemic diseases and systemic medication intake [3].

Rheumatoid Polyarthritis (RA) is a chronic inflammatory disease leading to arthritis, bursitis and tendovaginitis as a result of synovitis, which shows a progressive, but intermittent course eventually leading to joint destruction [4]. 
Hard and soft tissue injuries, pain, swelling and limited movement of joint are deemed prevalent complications of the problem. In order to relieve symptoms patient were obliged to use long term multiple medications including Methotrexate (MTX) and glucocorticoids (GC), sulfasalazine, nonsteroidal anti-inflammatory drug (NSAID), Remicade and so forth. Such combination of drugs may interfere with bone repair and osseointegration [5].

Moreover, RA is frequently accompanied by osteoporosis as a result of increased systemic bone turnover and anti-inflammatory and/or combined anti-immune treatment regimens [6].

Yet relatively little is known about the effect of such chronic medication use on the success of dental implants and osseointegration, long term effect of these medications have not been adequately investigated.

Facing this big controversy in the literature we are still asking whether dental implants are contraindicated in patients with RA and what are the survival rates.

This report discusses a successful 4 years follow up period of an immediate provisionalization of dental implant on a rheumatoid patient.

\section{Observation}

A 33-year-old woman was referred for the replacement of the teeth $\mathrm{N}^{\circ} 14$ lost after root fracture. The patient history revealed a Rheumatoid Arthritis diagnosed in 2014. According to the rheumatologist the patient has consumed $5 \mathrm{mg}$ of Prednisone daily and 15 $\mathrm{mg}$ to $20 \mathrm{mg}$ of Methotrexate onetime per week. The chief complaint of the patient was that the missing tooth was in the esthetic zone and she does accept no longer her removable prostheses.

On the CBCT we noticed adequate bone volume and density. After consultation with the rheumatologist and the consent of the patient we decide to suggest her for an implant with immediate provisional crown.

Premedication was performed, mouth rinsed with chlorhexidine $0.12 /$. Then a mucoperiosteal flap was released, a Neo CMI implant (Neo Biotech, Korea) of $4 \mathrm{~mm}$ diameter and $11.5 \mathrm{~mm}$ length was putted in healed bone with initial bone stability of more than $35 \mathrm{~N} / \mathrm{cm}$. Then a temporary abutment was connected to the implant and a provisional crown was confectioned with polycarboxyl- ate mold and provisional resin. The occlusion was checked and the crown was not loaded.

The postoperative care includes use of antibiotics (amoxicillin $500 \mathrm{mg}$ orally 3 times daily for 7 days) and an analgesic (paracetamol $500 \mathrm{mg}$ ). During the wound healing phase, the patient rinsed her mouth with chlorhexidine 0.12 /twice daily for 2 weeks. Sutures were removed 10 days after surgery (Figure 1).

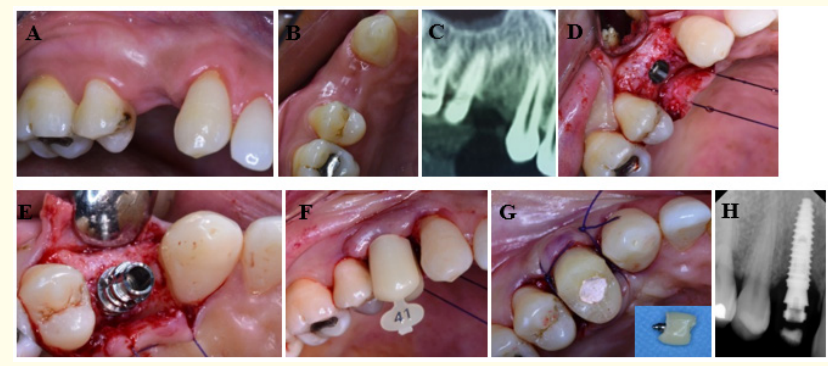

Figure 1: (A, B, C) Preoperative clinical and radiographic view of the site, (D) Implant placement, (E) connection of the temporary abutment, (F, G) Confection of the provisional crown and suture,

(H) Radiograph control 2 months after surgery.

After 6 months unloading phase, we checked osseointegration by performing clinical examination and X-ray observations. The provisional crown was well integrated with papilla regeneration. Finally, we took the impressions and the definitive crown was performed and cemented.

Clinical and radiological examinations were performed at implant and crown placement as well as at the follow-up visits after 6 , $12,24,36$ and 48 months.

Marginal bone levels were determined as the distance from the mesial and distal interproximal bone to the junction between the micro-threads and the machined bevel of the implant neck.

After 4 years of osseointegration, a 1 - $2 \mathrm{~mm}$ vertical bone loss was seen, the implant was stable with excellent osseointegration.

The patient's RA seems to have no effect on prognosis of implant. The treatment with methotrexate and glucocorticoid had not adversely affected the healing of the implant despite the antimetabolic and cytotoxic action of this medication. The peri-implant bone resorption was the same as that is normally expected (Figure 2). 

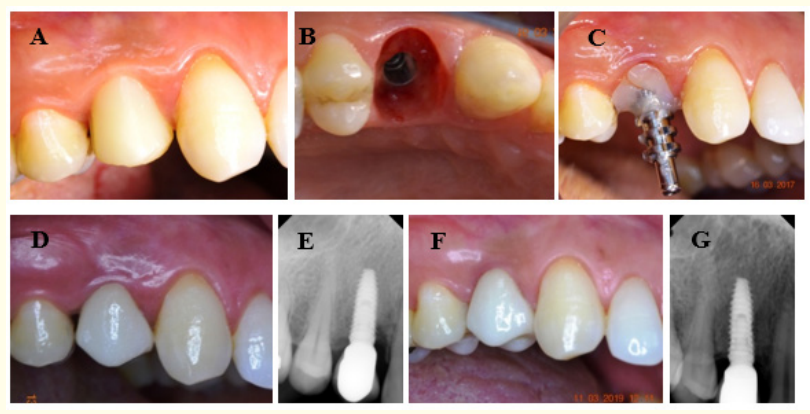

Figure 2: $(A, B)$ Healing around the temporary prosthesis one year after surgery with papilla regeneration, (C) Pick up impression, (D, E) clinical and radiographic conditions the day of prosthesis loading, (F, G) Clinical and radiographic conditions 4 years after surgery with interproximal bone stability and complete papilla regeneration.

\section{Discussion and Conclusion}

Success of osseointegration is an association of functional and aesthetic results and depends on some factors. In some cases, even when the patient had had appropriate bone quantity and quality and adequate clinical indications and recommendations are followed, failures in the osseointegration process still occur. This can be related to the immune-inflammatory host response which can alter bone repair and bone healing, ultimately leading to premature implant loss or peri-implant complication [7].

In this report RA seems to have had no effect on implant osseointegration and on the 3 years survival rate. The choice of an immediate provisionalization protocol the day of surgery didn't affect the bone remodeling and ameliorates the aesthetic outcomes.

There is a big controversy in the literature to award treatment with MTX; low dose MTX has a bone protecting effect by suppressing osteoclasts, adversely it eliminates the inflammatory reaction which is the first stage of osseointegration. That's why authors reported that MTX could interfere with the osseointegration process [8].

Annussek., et al. 2012 investigated in vitro study the effect of short time administration of low dose MTX on osteoblasts, as essential part of bone remodeling. They reported that the administration of MTX significantly reduced the proliferation of osteoblasts and mitochondrial activity [9]. This could be an important finding concerning mechanism affecting bone development, bone regen- eration and bone healing especially when restoring rheumatoid patients with implants.

Takavoli., et al. 2018 in an experimental study evaluated the short-time effects ( 4 weeks) of low-dose MTX on the osseointegration of dental implants in canine models. They concluded that low dose of MTX has the potential to interfere with the osseointegration process. The effects of MTX were investigated in this study without the presence of inflammatory diseases (RA) which reduce the ability to adopt the results for a human model. While this can be counted as a weak point of the study design, it can also be assumed as a strong point because the changes in the osseointegration process were literally related to the use of MTX [3].

Frequently MTX is associated with GC to treat RA. Long term $\mathrm{GC}$ administration has a significant effect on bone metabolism. The medication decreases the production of new osteoblasts precursors, causes early apoptosis of the mature osteoblasts and increases the life span of osteoclasts [8]. The results are systemic loss of bone mass, secondary osteoporosis and impaired bone healing.

It seems that there is a significant heterogeneity among the studies reviewed. Keller., et al. 2004, Carvas., et al. 2010, Almagro., et al. 2013 investigated bone to implant contact (BIC) on long bones animal models, they found reduced BIC [10].

However, in the study of Fujimoto 1998 [8] the BIC in the mandible of a rabbit model was significantly greater than that in tibia. This can be attributed to the embryological and structural differences between skeletal and jaw bones.

The unfavorable outcome obtained in many of the small sample-sized animal studies might not represent actual clinical situation in human patients [8].

Human studies on the subject are usually single patients [8]. Case series with long-term follow up are also limited.

In this report we notice that after four year follows up, the marginal bone loss didn't exceed $1 \mathrm{~mm}$, it was similar to that of healthy patients. Wound dehiscence or infection rates are not reported to be elevated due to the medication. The gingival parameters join a good aesthetic rendering.

Although the success of the implant supported prosthesis in patient with general predisposition and specific medicine intake, it does not permit general conclusion.

Citation: Hela Jegham., et al. "Four Year Follow Up of Immediate Provizionalization of Osseointegrated Dental Implant in Patient with Rheumatoid Polyarthritis". Acta Scientific Dental Sciences 4.10 (2020): 53-56. 
More better conducted human research and clinical studies are needed to fully determine the effects of these medications on implant osseointegration, and to reinforce our findings.

\section{Conflict of Interest}

All authors declare no conflict of interest, financial or otherwise.

\section{Bibliography}

1. AAP. "Glossary of Periodontal Terms". $4^{\text {th }}$ edition. Chicago, IL: The American Academy of Periodontology (2001): 28.

2. Albrektsson T and Wennerberg A. "On osseointegration in relation to implant surfaces". Clinical Implant Dentistry and Related Research 21 (2019): 4-7.

3. Tavakoli M., et al. "Evaluation of effect of low-dose methotrexate on osseointegration of implants: a biomechanical study on dogs". The Open Dentistry Journal 12 (2018): 546-554.

4. Haugeberg G. "Focal and generalized bone loss in rheumatoid arthritis: separate or similar concepts”. Nature Clinical Practice Rheumatology 4 (2008): 402-403.

5. Shokri M., et al. "Titanium implant osseointegration in rheumatoid arthritis patients: two case reports". Journal Dental School 35 (2017): 150-154.

6. Krennmair G., et al. "Dental implants in patients with rheumatoid arthritis: clinical outcome and peri-implant findings". Journal of Clinical Periodontology 37 (2010): 928-936.

7. Montes CC., et al. "Failing factors associated with osseointegrated dental implant loss". Implant Dentistry 16 (2007): 404412.

8. Petsinis V., et al. "The impact of glucocorticosteroids administered for systemic diseases on the osseointegration and survival of dental implants placed without bone grafting-A retrospective study in 31 patients". Journal of Cranio-Maxillofacial Surgery 45.8 (2017): 1197-1200.

9. Annussek T., et al. "Short time administration of antirheumatic drugs-methotrexate as a strong inhibitor of osteoblast's proliferation In vitro". Head and Face Medicine 8 (2012): 26.

10. Aghaloo T., et al. "The effects of systemic diseases and medications on implant osseointegration: a systematic review". The International Journal of Oral and Maxillofacial Implants 34 (2019): s35-s49.

\section{Assets from publication with us}

- Prompt Acknowledgement after receiving the article

- Thorough Double blinded peer review

- Rapid Publication

- Issue of Publication Certificate

- High visibility of your Published work

Website: www.actascientific.com/

Submit Article: www.actascientific.com/submission.php

Email us: editor@actascientific.com

Contact us: +919182824667 